\title{
Firehose constraints of the bi-Kappa-distributed electrons: a zero-order approach for the suprathermal electrons in the solar wind
}

\author{
M. Lazar, ${ }^{1,2}$ S. M. Shaaban, ${ }^{2,3 \star}$ S. Poedts ${ }^{2}$ and Š. Štverák ${ }^{4,5}$ \\ ${ }^{1}$ Institut für Theoretische Physik, Lehrstuhl IV: Weltraum- und Astrophysik, Ruhr-Universität Bochum, D-44780 Bochum, Germany \\ ${ }^{2}$ Center for Plasma Astrophysics, KU Leuven, Celestijnenlaan 200B, B-3001 Leuven, Belgium \\ ${ }^{3}$ Physics Department, Theoretical Physics Research Group, Faculty of Science, Mansoura University, 35516, Egypt \\ ${ }^{4}$ Institute of Atmospheric Physics, Czech Academy of Sciences, Bocni II 1401, 14131 Prague, Czech Republic \\ ${ }^{5}$ Astronomical Institute, Czech Academy of Sciences, Fričova 298, 25165 Ondřejov, Czech Republic
}

Accepted 2016 September 14. Received 2016 September 9; in original form 2016 March 27

\begin{abstract}
The increase of temperature predicted by the solar wind expansion in the direction parallel to the interplanetary magnetic field is already notorious for not being confirmed by the observations. In hot and dilute plasmas from space, particle-particle collisions are not efficient in constraining large deviations from isotropy, but the resulting firehose instability provides itself plausible limitations for the temperature anisotropy of both the electron and proton species. This paper takes into discussion the suprathermal (halo) electrons, which are ubiquitous in the solar wind, and may be highly anisotropic and susceptible to the firehose instability. Suprathermals enhance the high-energy tails of the velocity distributions making them well described by the Kappa distribution functions, with the advantage that these are power laws suitable to reproduce either the entire distribution or only the suprathermal halo tails. New features of the instability are captured from a linear stability analysis of bi-Kappa-distributed electrons with the temperature depending on the power-index $\kappa$. This approach enables a realistic interpretation of non-thermal electrons and their effects on the instability: growth rates are systematically stimulated and thresholds are lowered with decreasing the power-index $\kappa$. In a zero-order limiting approach of the halo component (minimizing the effects of a cooler and less anisotropic core population), the instability thresholds align to the limits of the temperature anisotropy reported by the observations. These results provide new and valuable support for an extended implication of the firehose instability in the relaxation of temperature anisotropy in collisionless plasmas from space.
\end{abstract}

Key words: instabilities-plasmas-methods: analytical-methods: observational-solar wind.

\section{INTRODUCTION}

In collisionless plasmas from space, large deviations from thermodynamic equilibrium cannot be relaxed by the particle-particle (Coulomb) collisions, but can presumably be constrained by the resulting kinetic instabilities. Thus, if the solar wind expands adiabatically, the CGL (Chew, Goldberger \& Low 1956) invariants conserve leading to an indefinite increase of temperature $(T)$ in the direction parallel to the interplanetary magnetic field, i.e. $T_{\|}>$ $T_{\perp}$, where $\|$ and $\perp$ denote directions relative to the magnetic field. However, the in situ measurements do not confirm such an increase of the parallel temperature with heliocentric distance, but indicate bounds of the temperature anisotropy of plasma particles (Kasper,

^E-mail: shaaban.mohammed@student.kuleuven.be
Lazarus \& Gary 2002; Hellinger et al. 2006; Štverák et al. 2008). Because collisions are not efficient, the most invoked mechanism that can limit the increase of parallel temperature is the firehose instability (Eviatar \& Schulz 1970; Kasper et al. 2002; Hellinger et al. 2006; Štverák et al. 2008; Lazar et al. 2014b). For anisotropies exceeding the instability thresholds, the free energy is dissipated by the resulting growing fields, which may also scatter particles back towards quasi-equilibrium states and prevent the anisotropy to grow (Gary \& Lee 1994; Gary et al. 1998).

Driven by the anisotropic electrons with an excess of parallel temperature $A \equiv T_{\perp} / T_{\|}<1$, the electron firehose instability (EFHI) may play an important role in mediating the (free) energy transfer from anisotropic electrons to protons (Paesold \& Benz 1999; Messmer 2002). This energy transfer from small to large scales is facilitated by the quasi-parallel EFH modes, which are left-handed (LH) circularly polarized and have characteristic frequencies and growth rates 
in the range of the proton cyclotron frequency. Besides the periodic (non-zero frequency) modes predominant in direction parallel to the magnetic field, the same firehose mechanism may destabilize an additional aperiodic branch that exists only for oblique directions (Gary \& Nishimura 2003; Camporeale \& Burgess 2008; Hellinger et al. 2014).

Although the suprathermal (halo) electrons are a constant presence in the solar wind (Lin 1998; Pierrard \& Lazar 2010; Lazar, Schlickeiser \& Poedts 2012), enhancing the high-energy tails of their distributions, the anisotropic temperature is in general quantified by a bi-Maxwellian distribution function, which is relevant for the thermal core but cannot describe the suprathermal tails of the observed distributions. Instead, these suprathermal tails are well reproduced by the Kappa distribution function (Vasyliunas 1968; Maksimovic et al. 2005; Pierrard \& Lazar 2010), which is nearly Maxwellian at low energies and decreases as a power law at high energies (Meyer-Vernet 2007). A Kappa power law can therefore be applied in two distinct ways, either as global model to incorporate both the thermal core and the suprathermal halo, or to reproduce only the suprathermal tails separate from the core of the distribution (Leubner 2004; Maksimovic et al. 2005; Štverák et al. 2008). Modelling the entire distribution with a global Kappa involves a lower number of parameters, and it is therefore preferred in observational analyses (Vasyliunas 1968; Collier et al. 1996; Maksimovic, Pierrar \& Riley 1997) as well as theoretical predictions, see reviews by Hellberg, Mace \& Cattaert (2005) and Pierrard \& Lazar (2010). Moreover, Leubner (2004) has shown that core-halo structures are a natural ingredient of non-extensive systems and at the same time global Kappa distribution functions turn out as consequence of non-extensive statistics for such systems subject to long-range interactions as solar wind plasmas. Complex models combining a bi-Maxwellian core and a bi-Kappa halo are difficult to manipulate, but may be powerful in finding out details about these two components (Maksimovic et al. 2005; Štverák et al. 2008). Thus, statistical diagrams obtained for the temperature anisotropies of these two components can be used to contrast with the instability thresholds predicted by the linear theory. From a bi-Maxwellian approach of the FHI, the aperiodic mode is found to grow faster with thresholds approaching well enough the limits of the core anisotropy (Štverák et al. 2008). For the suprathermal halo, the anisotropy limits are markedly departed from the instability thresholds predicted by a bi-Maxwellian, see fig. 6 and the analysis in Štverák et al. (2008). To resolve this disagreement, the instability conditions must be derived for the same bi-Kappa model invoked in the parametrization of suprathermal electrons.

The bi-Kappa model is extensively invoked in theories of wave dispersion and stability by adopting two alternative assumptions for the temperature of Kappa populations to be either dependent or independent of the power-index $\kappa$. The existing studies of the FHI (Lazar \& Poedts 2009; Lazar, Poedts \& Schlickeiser 2011) assume $\kappa$-independent temperatures, and find, contrary to the expectations, that the instability is inhibited by the suprathermals (i.e. with decreasing the power-index $\kappa$ ) and the instability thresholds do not approach but depart even more from the anisotropy bounds of the solar wind suprathermal electrons. However, from a recent analysis on the applicability of Kappa distributions (Lazar, Poedts \& Fichtner 2015b; Lazar, Fichtner \& Yoon 2016), it becomes evident that a representation with a $\kappa$-dependent temperature may provide a more natural interpretation of the suprathermal populations for three fundamental reasons: (1) it corresponds to a Maxwellian limit that reproduces more accurately the thermal core of the distribution enabling for a direct and realistic comparison (Lazar et al. 2015b); (2) the kinetic instabilities show a systematic stimulation in the presence of suprathermal electrons (Lazar et al. 2015b; Viňas et al. 2015; Shaaban et al. 2016b) as one may expect from the excess of free energy accumulated by these populations; and (3) the observations show strong evidence of $\kappa$-dependent temperatures, which increase in the presence of suprathermal populations, i.e. temperatures increase with decreasing the power-index $\kappa$ (Pierrard et al. 2016). Such Kappa models with $\kappa$-dependent temperatures have been introduced by Leubner \& Schupfer $(2000,2001)$ in a series of studies of the kinetic mirror instability, which is driven by an opposite anisotropy, i.e. an excess of perpendicular temperature $T_{\perp}>T_{\|}$.

Motivated by these premises, in this paper we propose a refined analysis of the EFHI by modelling the anisotropic electrons with a bi-Kappa distribution function with $\kappa$-dependent temperatures. Our present analysis restricts to the same parallel (non-zero frequency) modes studied before by Lazar \& Poedts (2009) and Lazar et al. (2011) for a plasma of isotropic protons and anisotropic electrons (with $T_{\mathrm{e}, \|}>T_{\mathrm{e}, \perp}$ ). In Section 2, we introduce the velocity distribution functions assuming Maxwellian protons and bi-Kappadistributed electrons, and derive the dispersion relation for the FHI modes. The main features of the instability, e.g. growth rates, wavefrequency, the unstable wavenumbers, and the anisotropy thresholds, are examined in Section 3. These results are intended to provide a straightforward characterization of the EFHI driven by the anisotropic electrons, when these are well described by a global bi-Kappa distribution function. In this case, a confrontation of the instability thresholds with the observations in space plasmas is not possible because statistical diagrams of temperature anisotropy versus plasma beta (determined with a global Kappa) do not exist. However, thresholds predicted by our (bi-)Kappa approach are compared in Section 3.2 with the anisotropy limits of the solar wind halo electrons, considering our simplified model (with only one electron component) as a zero-order approach that captures only the kinetic effects of the suprathermal halo and minimizes the influence of the thermal core (usually cooler and less anisotropic than the halo). Further contrast is provided with the results obtained by Štverák et al. (2008), who invoked a similar zero-order approach to compare the same observations with the less realistic thresholds predicted by a bi-Maxwellian model. The results of this work are discussed and summarized in Section 4.

\section{BI-KAPPA ELECTRONS. DISPERSION RELATION}

We first introduce the analytical model for the velocity distributions of an electron-proton plasma, typical for the solar wind conditions in the absence of energetic events (e.g. fast winds or coronal mass ejections). The particle distributions may be assumed gyrotropic, i.e. isotropic in the plane transverse to the magnetic field, with a bi-axis temperature anisotropy $T_{\perp} \neq T_{\|}$, where $\|$and $\perp$ denote directions relative to the magnetic field. In the absence of beams, suprathermal populations enhance the tails of velocity distributions, and in velocity space with polar coordinates $\left(v_{\perp} \cos \phi, v_{\perp} \sin \phi, v_{\|}\right)$ $=\left(v_{x}, v_{y}, v_{z}\right)$ these distributions are well reproduced by the family of bi-Kappa distribution functions (see, for instance, the reviews by Pierrard \& Lazar 2010; Lazar et al. 2012)

$$
F^{\kappa}\left(v_{\|}, v_{\perp}\right)=\frac{1}{\pi^{3 / 2} \theta_{\|} \theta_{\perp}^{2}} \frac{\Gamma[\kappa]}{\kappa^{1 / 2} \Gamma[\kappa-1 / 2]}\left(1+\frac{v_{\|}^{2}}{\kappa \theta_{\|}^{2}}+\frac{v_{\perp}^{2}}{\kappa \theta_{\perp}^{2}}\right)^{-\kappa-1},
$$


which is normalized to unity, and where $\theta_{\|, \perp}$ are thermal velocities defined by, respectively, the parallel and perpendicular temperatures as moments of second order

$$
\begin{aligned}
T_{\|}^{\kappa} & =\frac{m}{k_{B}} \int \mathrm{d} \boldsymbol{v} v_{\|}^{2} F^{\kappa}\left(v_{\|}, v_{\perp}\right)=\frac{\kappa}{\kappa-3 / 2} \frac{m \theta_{\|}^{2}}{2 k_{B}}, \\
T_{\perp}^{\kappa} & =\frac{m}{2 k_{B}} \int \mathrm{d} \boldsymbol{v} v_{\perp}^{2} F^{\kappa}\left(v_{\|}, v_{\perp}\right)=\frac{\kappa}{\kappa-3 / 2} \frac{m \theta_{\perp}^{2}}{2 k_{B}} .
\end{aligned}
$$

The bi-Kappa simply reduces to a bi-Maxwellian, used in Štverák et al. (2008), only in the limit of a very large $\kappa \rightarrow \infty$

$F^{M}\left(v_{\|}, v_{\perp}\right)=\frac{1}{\pi^{3 / 2} \theta_{\|} \theta_{\perp}^{2}} \exp \left(-\frac{v_{\|}^{2}}{\theta_{\|}^{2}}+\frac{v_{\perp}^{2}}{\theta_{\perp}^{2}}\right)$,

with

$$
\begin{aligned}
& T_{\|}^{M}=\frac{m}{k_{B}} \int \mathrm{d} \boldsymbol{v} v_{\|}^{2} F^{M}\left(v_{\|}, v_{\perp}\right)=\frac{m \theta_{\|}^{2}}{2 k_{B}}<T_{\|}^{\kappa}, \\
& T_{\perp}^{M}=\frac{m}{2 k_{B}} \int \mathrm{d} \boldsymbol{v} v_{\perp}^{2} F^{M}\left(v_{\|}, v_{\perp}\right)=\frac{m \theta_{\perp}^{2}}{2 k_{B}}<T_{\perp}^{\kappa} .
\end{aligned}
$$

Notice in this case that the temperature of Kappa electrons decreases with increasing the power-index $\kappa$ and reaches a minimum for the Maxwellian limit. Leubner \& Schupfer $(2000,2001)$ have originally invoked such Kappa models with $\kappa$-dependent temperatures to investigate the instability conditions for the kinetic mirror modes. The parallel plasma beta parameter becomes a function of power-index $\kappa$

$\beta_{\mathrm{e}, \|}^{K}(\kappa)=\frac{8 \pi n_{\mathrm{e}} k_{B} T_{\mathrm{e}, \|}^{K}}{B_{0}^{2}}=\frac{2 \kappa}{2 \kappa-3} \beta_{\mathrm{e}, \|}^{M}>\beta_{\mathrm{e}, \|}^{M}$.

In the direction parallel to the magnetic field $(\boldsymbol{k} \| \boldsymbol{B})$, the electromagnetic (EM) modes are decoupled from the electrostatic oscillations, and are described by the following linear dispersion relation (Gary 1993)

$$
\begin{aligned}
\frac{k^{2} c^{2}}{\omega^{2}}= & 1+\frac{4 \pi}{\omega^{2}} \sum_{\mathrm{a}} \frac{e_{\mathrm{a}}}{m_{\mathrm{a}}} \int_{-\infty}^{\infty} \frac{\mathrm{d} v_{\|}}{\omega-k v_{\|} \pm \Omega_{a}} \int_{0}^{\infty} \mathrm{d} v_{\perp} \\
& \times v_{\perp}^{2}\left[\left(\omega-k v_{\|}\right) \frac{\partial F_{\mathrm{a}}}{\partial v_{\perp}}+k v_{\perp} \frac{\partial F_{\mathrm{a}}}{\partial v_{\|}}\right],
\end{aligned}
$$

where $\omega$ and $k$ are, respectively, the frequency and the wavenumber of the plasma modes, $c$ is the speed of light in vacuum, $\Omega_{\mathrm{a}}=q_{\mathrm{a}} B_{0} /\left(m_{\mathrm{a}} c\right)$ is the gyrofrequency for the particles of sort $a$, e.g. $a=e$ for electrons and $a=p$ for protons, respectively, and ' \pm ' describes the circularly polarized EM modes with right-handed (RH) and LH polarizations, respectively. For the model introduced in equation (1), the dispersion relation becomes

$$
\begin{aligned}
\frac{k^{2} c^{2}}{\omega^{2}}= & 1+\sum_{\mathrm{a}} \frac{\omega_{\mathrm{a}, h}^{2}}{\omega^{2}}\left[A_{\mathrm{a}}-1\right. \\
& \left.+\frac{\left(A_{\mathrm{a}}-1\right)\left(\omega \pm \Omega_{\mathrm{a}}\right)+\omega}{k \theta_{\mathrm{a}, \|}} Z_{\kappa}\left(\frac{\omega \pm \Omega_{\mathrm{a}}}{k \theta_{\mathrm{a}, \|}}\right)\right],
\end{aligned}
$$

where $A_{\mathrm{a}}=T_{\mathrm{a}, \perp} / T_{\mathrm{a}, \|}$ is the temperature anisotropy,

$$
\begin{aligned}
Z_{\kappa}(f)= & \frac{1}{\pi^{1 / 2} \kappa^{1 / 2}} \frac{\Gamma(\kappa)}{\Gamma\left(\kappa-\frac{1}{2}\right)} \\
& \times \int_{-\infty}^{\infty} \mathrm{d} x \frac{\left(1+x^{2} / \kappa\right)^{-\kappa}}{x-f}, \quad \Im(f)>0,
\end{aligned}
$$

is the Kappa plasma dispersion function (Lazar, Schlickeiser \& Shukla 2008) of argument

$f_{\kappa}=\frac{\omega \pm \Omega_{\mathrm{a}}}{k \theta_{\mathrm{a}, \|}}$.

In the Maxwellian limit, this function reduces to the standard plasma dispersion function (Fried \& Conte 1961)

$Z(f)=\frac{1}{\pi^{1 / 2}} \int_{-\infty}^{+\infty} \mathrm{d} x \frac{\exp \left(-x^{2}\right)}{x-f}, \quad \Im(f)>0$,

of argument

$f=\frac{\omega \pm \Omega_{\mathrm{a}}}{k w_{\mathrm{a}}}$.

Note that for our model introduced in equations (1)-(6), the anisotropy does not depend on $\kappa$, i.e. $A=T_{\perp}^{\kappa} / T_{\|}^{\kappa}=T_{\perp}^{M} / T_{\|}^{M}$.

We investigate the EFHI, which is an LH EM mode driven unstable by an excess of electron temperature in parallel direction $T_{\mathrm{e}, \|}>$ $T_{\mathrm{e}, \perp}$, i.e. $A_{\mathrm{e}}<1$. According to equation (9), the dispersion relation describing these modes can be rewritten with normalized quantities as follows

$$
\begin{aligned}
& \mu\left[A_{\mathrm{e}}-1+\frac{A_{\mathrm{e}}(\tilde{\omega}+\mu)-\mu}{\tilde{k} \sqrt{\mu \beta_{\mathrm{e}, \|}^{M}}} Z_{\kappa}\left(\frac{\tilde{\omega}+\mu}{\tilde{k} \sqrt{\mu \beta_{\mathrm{e}, \|}^{M}}}\right)\right] \\
& +\frac{\tilde{\omega}}{\tilde{k} \sqrt{\beta_{\mathrm{e}, \|}^{M} / \Theta}} Z\left(\frac{\tilde{\omega}-1}{\tilde{k} \sqrt{\beta_{\mathrm{e}, \|}^{M} / \Theta}}\right)=\tilde{k}^{2},
\end{aligned}
$$

where protons are assumed Maxwellian and isotropic $A_{\mathrm{p}}=1$, and $\tilde{\omega}=\omega / \Omega_{\mathrm{p}}, \tilde{k}=k c / \omega_{\mathrm{p}, \mathrm{p}}, \mu=m_{\mathrm{p}} / m_{\mathrm{e}}$ is the proton/electron mass ratio, $\Theta=T_{\mathrm{e}, \|}^{M} / T_{\mathrm{p}, \|}^{M}$ is the electron/proton parallel temperature ratio, and $\beta_{\mathrm{e}, \|}^{M}=8 \pi n_{\mathrm{e}} k_{B} T_{\mathrm{e}, \|}^{M} / B_{0}^{2}$ is the parallel electron beta parameter in the Maxwellian limit $\kappa \rightarrow \infty$. The dispersion relation for biMaxwellian-distributed electrons is obtained from equation (14) by changing $Z_{\kappa}$ with the Maxwellian plasma dispersion function from equation (12).

\section{EFHI SOLUTIONS}

We have solved the dispersion relation (equation 14) numerically, and analysed the unstable firehose solutions for a wide variety of plasma regimes susceptible to this instability. In this section, we present the main features of the EFHI, namely, growth rates, wavefrequencies, and wavenumbers, as well as the anisotropy thresholds, and restrict our discussions only to a number of representative cases. However, the anisotropy thresholds displayed and analysed in the second part of this section cover extended conditions encountered in space plasmas, for instance, in the solar wind and planetary magnetospheres. Since only the electrons are anisotropic, in the next analysis we omit the labelling subscript 'e', for the anisotropy, $A$, and the plasma beta parameter, $\beta_{\|}$. The effects of Kappa-distributed electrons are triggered by their temperature anisotropy and the abundance of suprathermal populations, which is quantified by the finite (low) values of the power-index $\kappa$.

\subsection{Unstable solutions}

First, we examine the growth rates and the wave-frequency of the EFHI for different plasma regimes conditioned in principal by the parallel plasma beta parameter $\beta_{\|}$, the electron anisotropy $A$, and the power-index $\kappa$. The regimes identified in Figs 1 and 2 are 

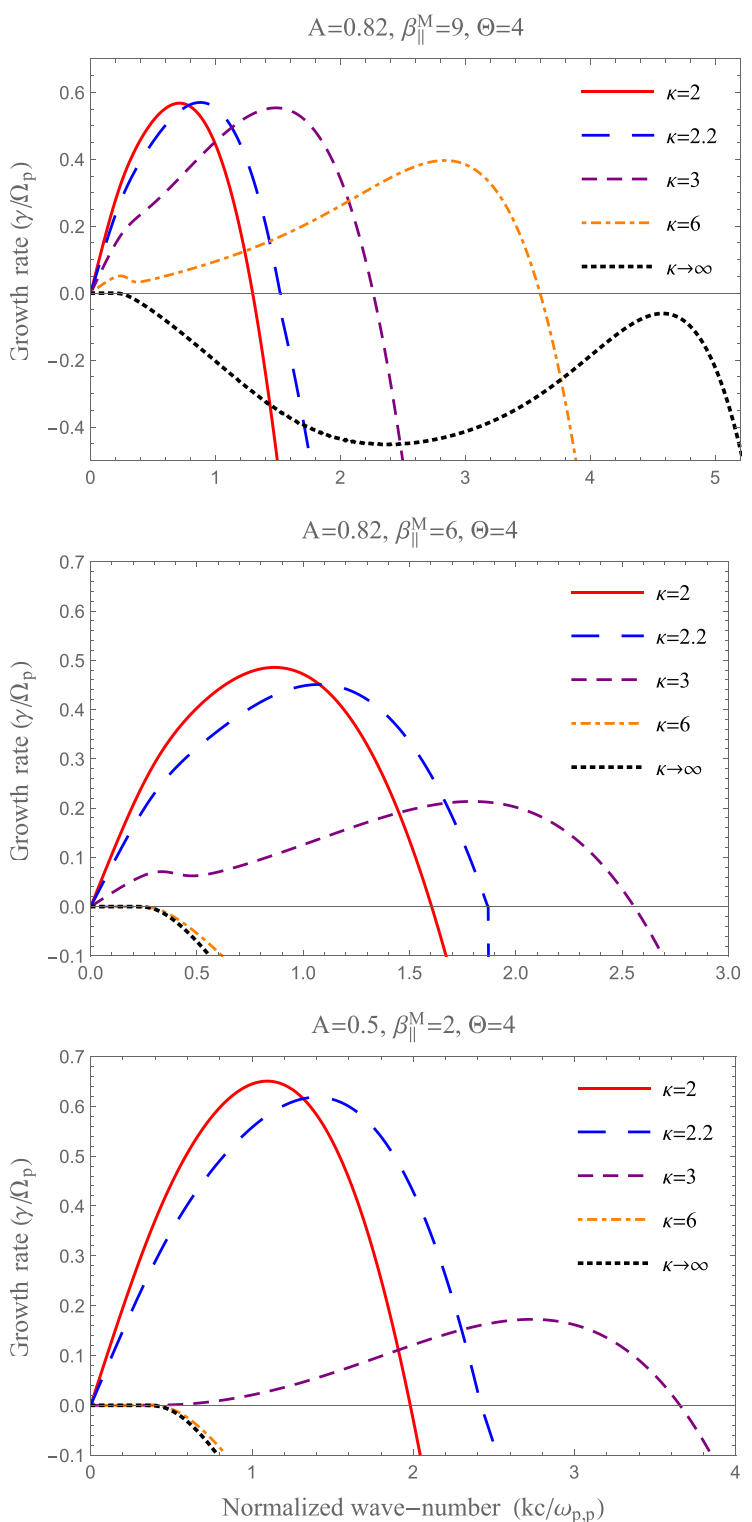

Figure 1. Effects of the suprathermal electrons quantified by the powerindex $\kappa=2,2.2,3,6, \infty$, on the growth rates of EFHI for different plasma beta explicitly given in each panel. Here $\beta_{\|} \equiv \beta_{\|}^{M}$ is the one used in equation (14).

specific to the firehose instability, when a magnetized plasma becomes penetrable by the LH EM fluctuations propagating parallel to the magnetic field with frequencies higher than the proton cyclotron frequency. All the unstable modes, i.e. with $\gamma>0$ in Fig. 1, exhibit this property that becomes evident in Fig. 2, where their wavenumber dispersion extends to high frequencies exceeding $\Omega_{\mathrm{p}}$. Increasing the presence of suprathermals, i.e. lowering the powerindex $\kappa$, the range of unstable wavenumbers is restrained, but the wave-frequencies and the instability growth rates are enhanced. These effects are in general stimulated by increasing the plasma beta parameter $\beta_{\|}$, the temperature anisotropy and the electronproton temperature contrast $\Theta$. Plots evidencing the influence of $\Theta$ are not shown here, being less relevant for the scope of this paper. The unstable solutions displayed in Figs 1-4 are obtained for the same value of this parameter, namely, for $\Theta=4$ in accordance to the observations in the slow solar wind (Newbury et al. 1998).
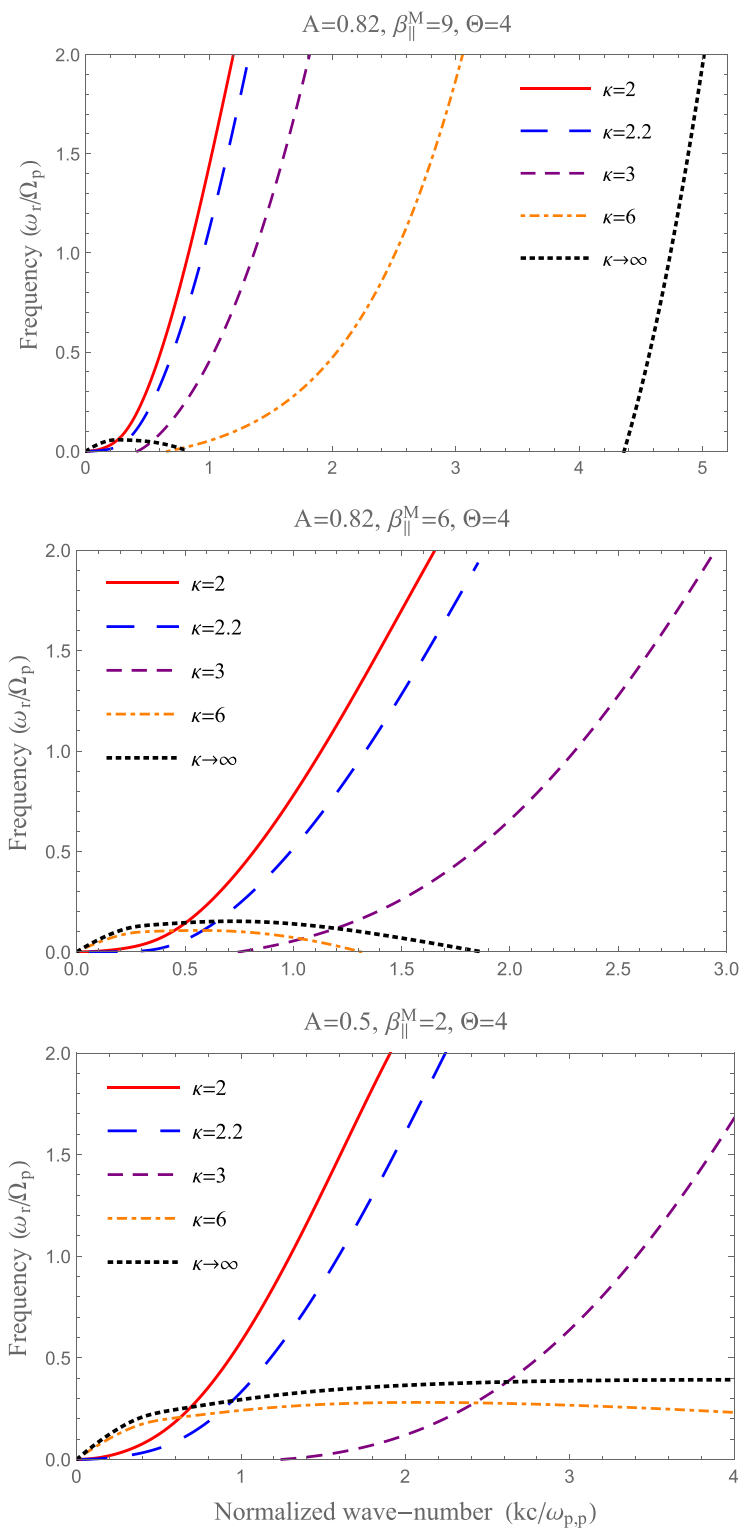

Figure 2. Effects of the suprathermal electrons $(\kappa=2,2.2,3,6, \infty)$ on the wave-frequency of EFHI for the same cases considered in Fig. 1.

At higher values of $\kappa$, the instability conditions may be not satisfied and the EM modes are damped, e.g. $\gamma<0$ for $\kappa \geq 6$ in Fig. 1, middle and bottom panels. For these modes, the wave-frequency dispersion curves displayed in Fig. 2 have a different allure, showing an asymptotic increase similar to the ion (proton) cyclotron modes with frequencies always smaller than $\Omega_{\mathrm{p}}$. These are LH modes damped by the protons and limited only to the large (proton) scales. At lower scales controlled by the electrons (higher wavenumbers), these modes change (mode conversion) to RH polarization (i.e. the wave-frequency displayed in Fig. 2 becomes negative) that is specific to the electron whistlers.

In Figs 3 and 4, we show that these LH-polarized modes with a wavenumber dispersion resembling that of the electromagnetic ion cyclotron (EMIC) modes can be destabilized by the anisotropic bi-Kappa-distributed electrons, see middle and bottom panels. This is a new regime of the EFHI destabilizing only the low-frequency branch of the LH modes with wave-frequency showing an asymptotic increase of their wave-frequencies and remaining always below 

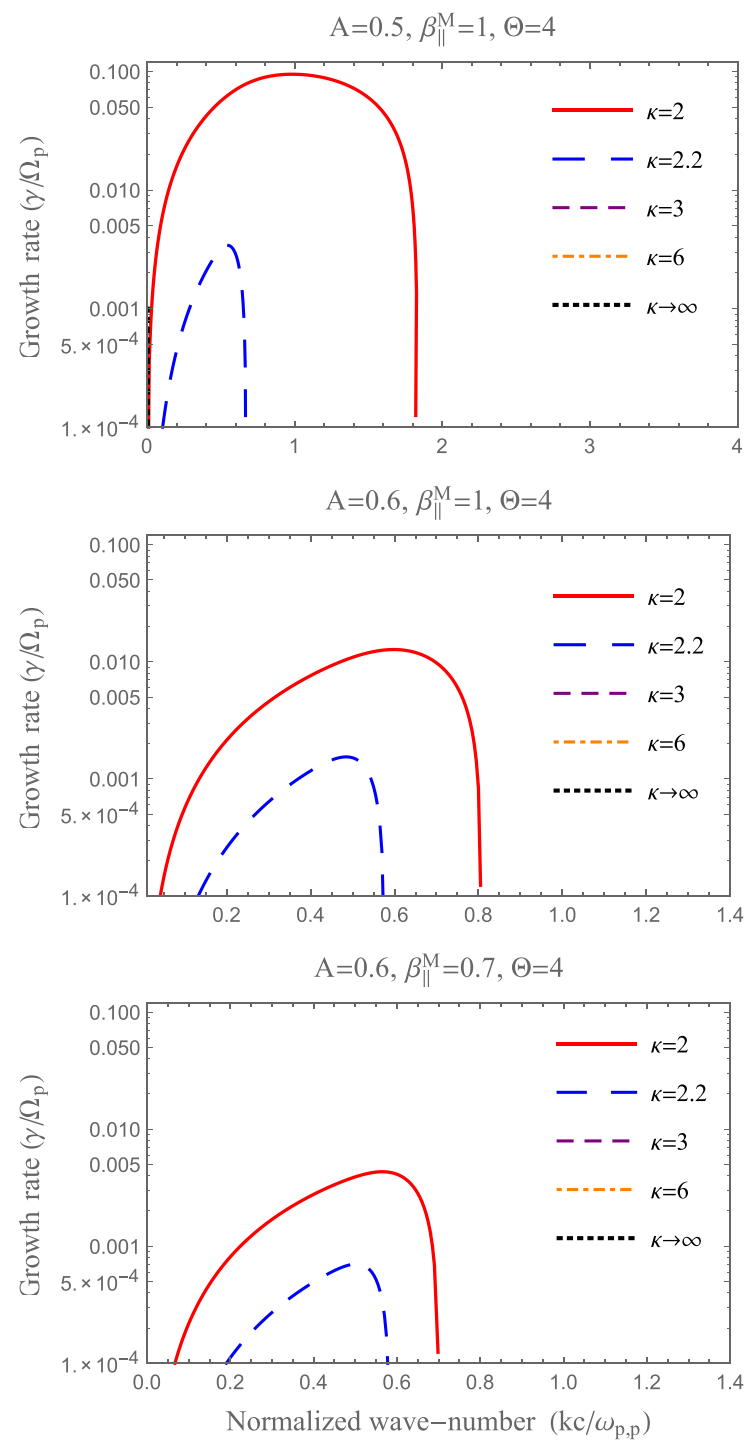

Figure 3. Effects of the suprathermal electrons quantified by the powerindex $\kappa=2,2.2,3,6, \infty$, on the growth rates of EFHI for a lower $\beta_{\|}^{M}=0.6$.

$\Omega_{\mathrm{p}}$. To establish this regime, the kinetic effects of the electrons are also tempered by considering lower values of plasma beta, but then the instability is triggered only by the anisotropic distributions with sufficiently low $\kappa$, e.g. $\kappa<3$ in Figs 3 and 4. Furthermore, in this case, both the (maximum) growth rates and the range of the unstable wavenumbers are considerably enhanced by increasing the presence of suprathermals, i.e. lowering the values of $\kappa$. Again, these features seem to be more specific to the instability of the cyclotron modes (Shaaban et al. 2016b). The transition between the classical EFH solutions (exemplified in Figs 1 and 2) and the new regime of a lowfrequency EFHI is suggestively shown in Figs 3 and 4, top panels. In these panels, we have displayed unstable solutions specific to both these two regimes: the solid-line solution obtained for $\kappa=2$ is a classical firehose, while the next long-dashed-line solution obtained for $\kappa=2.2$ is already more specific to the new regime of EFHI. In this case, it is only the power-index $\kappa$ that may switch between these two regimes, but an extended direct comparison of the other EFH solutions in Figs 1-4 clearly shows that these regimes are also conditioned by the temperature anisotropy and the plasma beta.
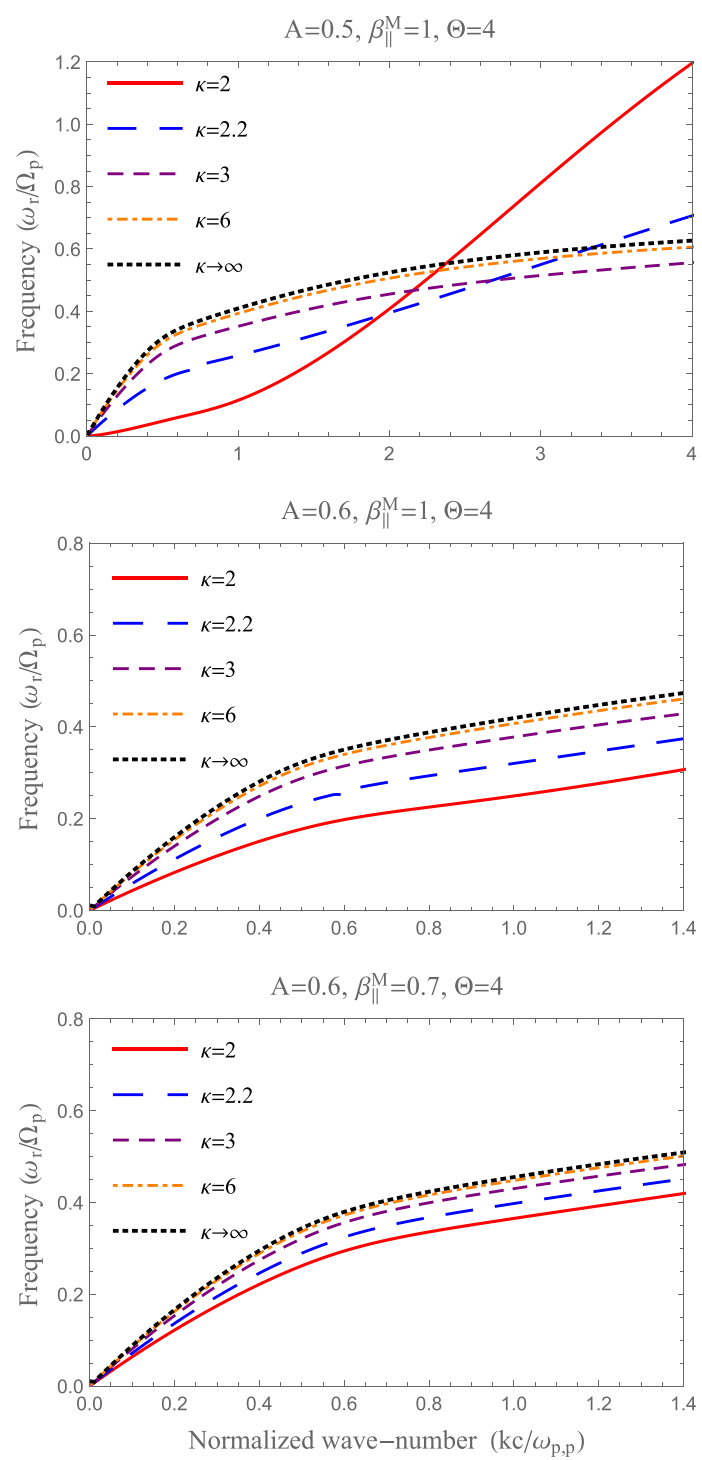

Figure 4. Effects of the suprathermal electrons $(\kappa=2,2.2,3,6, \infty)$ on the wave-frequency of EFHI for the same cases considered in Fig. 3.

\subsection{Thresholds: predictions versus observations}

In the second part of this section, we analyse the anisotropy thresholds of the instability. These thresholds represent plasma conditions associated with given values of the maximum growth rate, usually small values, e.g. $\gamma_{\mathrm{m}} / \Omega_{\mathrm{p}}=10^{-2}, 10^{-3}$, approaching the marginal stability $\gamma_{\mathrm{m}} / \Omega_{\mathrm{p}} \rightarrow 0$. In Fig. 5, we display the instability thresholds associated with $\gamma_{\mathrm{m}} / \Omega_{\mathrm{p}}=10^{-3}$ and derived for different values of the electron power-index $\kappa$. These are isocontours of the electron temperature anisotropy, $A$, as a function of the parallel electron plasma beta $\beta_{\|}$, fitted to an inverse correlation law of the form (Gary \& Lee 1994; Gary et al. 1998)

$A=1-\frac{a}{\beta_{\|}^{b}}$.

The values obtained for the fitting parameters $a$ and $b$ are given in Table 1 . Higher values of $\beta_{\|}$, associated with hotter plasmas or less intense magnetic fields, imply lower deviations from isotropy to trigger the instability. For the plasma beta parameter, we consider an extended range of values $0.1<\beta_{\|}<50$ 

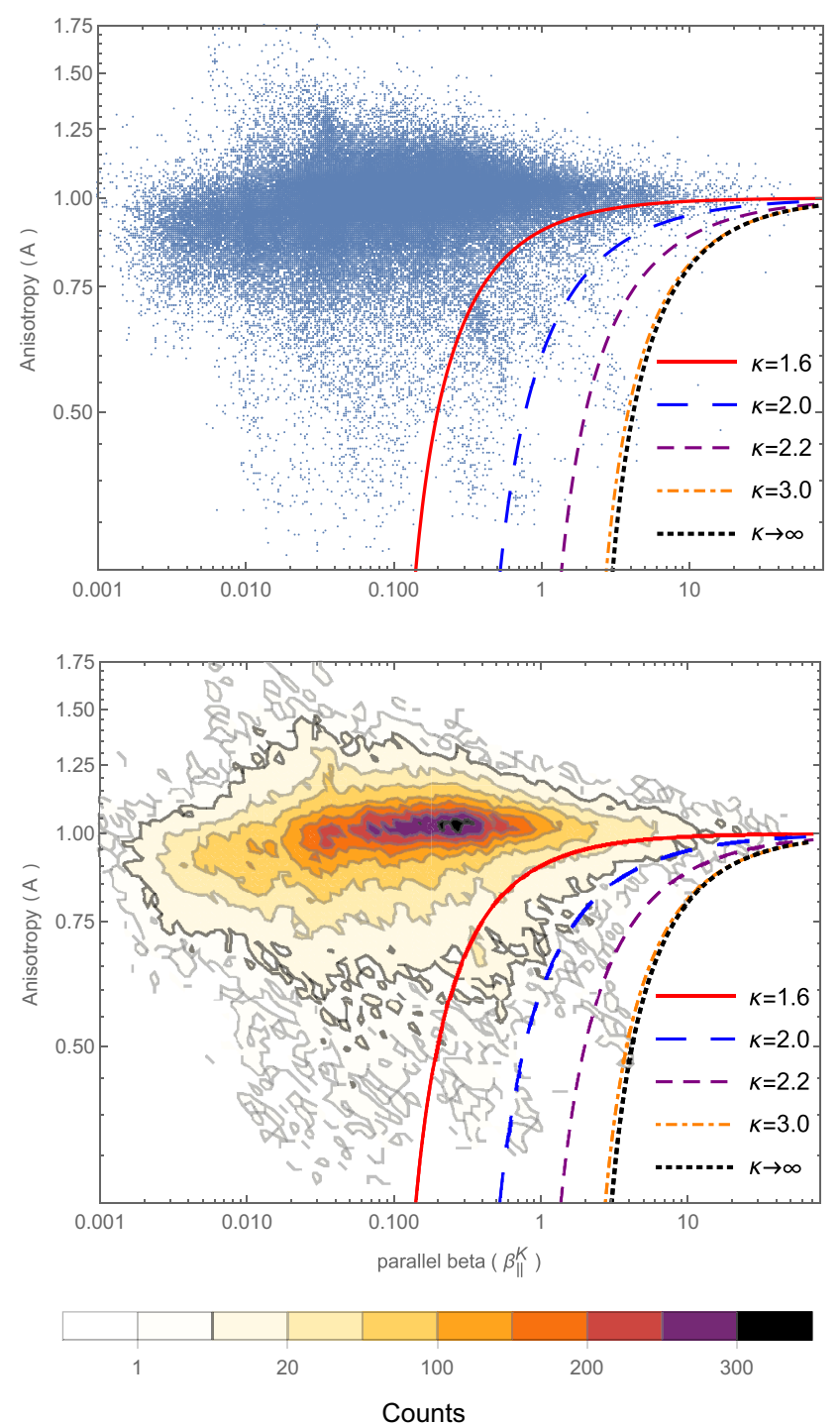

Figure 5. Comparison of the anisotropy thresholds (equation 15) for maximum growth rates $\gamma_{\mathrm{m}} / \Omega_{\mathrm{p}}=10^{-3}$ with the electron halo temperature anisotropy measured in the solar wind and displayed using a scatter plot data in the top panel and a histogram data in the bottom panel. The anisotropy is plotted versus the halo parallel plasma beta $\beta_{\|}^{K}$ defined in equation (7).

Table 1. Fitting parameters for thresholds $\gamma_{\mathrm{m}} / \Omega_{\mathrm{p}}=10^{-3}$.

\begin{tabular}{lccccc}
\hline Fit & $\kappa=1.6$ & $\kappa=2$ & $\kappa=2.2$ & $\kappa=3$ & $\kappa \rightarrow \infty$ \\
\hline$a$ & 0.0999 & 0.4001 & 0.9256 & 1.9226 & 2.2303 \\
$b$ & 0.9933 & 0.8708 & 0.8996 & 1.0004 & 1.0457 \\
\hline
\end{tabular}

relevant for the electrons in space plasma (Štverák et al. 2008). The effects of suprathermal electrons is reconfirmed here by a systematic stimulation of the (maximum) growth rates with decreasing $\kappa$. As a consequence, the anisotropy thresholds are found to be markedly lowered in the presence of suprathermals, and this effect may be enhanced by increasing the temperature contrast between electrons and protons (not shown here). Larger variations of the anisotropy thresholds are obtained at lower values of $\kappa$. In the absence of collisions, the EFHI is expected to constrain the temperature anisotropy in the solar wind, with thresholds ap- proaching the anisotropy limits reported by the observations. Unfortunately, statistical diagrams of temperature anisotropy versus plasma beta determined with a global bi-Kappa model do not exist, making impossible a direct confrontation with our instability thresholds.

At this stage, we can compare the instability thresholds with the anisotropy of the halo suprathermal electrons detected in the solar wind and parametrized with the same bi-Kappa model. As shown in the Introduction, a bi-Kappa can be used to describe either the entire distribution, or only the suprathermal halo component. To compare our results with the observations of the halo electrons, our bi-Kappa approach must be considered as a zero-order approach that captures only the effects of the halo component and minimizes the influence of the thermal core. The observational data for the halo electrons, namely their anisotropy versus their parallel beta, are displayed in Fig. 5 as a scatter plot in the top panel, and as a histogram, counting the number of events within a colour logarithmic scale, in the bottom panel. The data set is selected from more than 120000 events detected by three space missions, Helios 1, Cluster II, and Ulysses, at different heliocentric distances (in the interval 0.3-3.95 au) in the ecliptic. In order to neglect the effects of the strahl, which is not taken into account in our present study, the observational data in Fig. 5 are selected for specific conditions, for instance, in the slow winds (with bulk speeds less than $500 \mathrm{~km} \mathrm{~s}^{-1}$ ), and beyond 1 au where the density of the strahl population is significantly diminished by comparison to the non-streaming halo population (Maksimovic et al. 2005). More details about the electron analyzers on spacecraft, and the methods of correction and reconstruction of the $3 \mathrm{D}$ velocity distributions can be found in Štverák et al. (2008). These authors have used the same set of events to analyse the temperature anisotropy of the main electron populations, namely, the thermal core and suprathermal halo, and the most plausible constraints exercised on their temperature anisotropy by different physical mechanisms, e.g. collisions and kinetic instabilities.

From a detailed investigation of the electron core anisotropy, Štverák et al. (2008) have found that particle-particle collisions (from early ages in the solar corona) still may have an effect constraining low levels of anisotropy, while the kinetic instabilities occur for larger deviations from isotropy exceeding their thresholds. Indeed, the instability thresholds provided by a bi-Maxwellian model were found to shape well the limits of the core anisotropy, but not the limits of the halo anisotropy. This disagreement is evident in fig. 6 from Štverák et al. (2008), which compares the observed halo anisotropy measured with a bi-Kappa and the instability thresholds provided by a zero-order approach that assumes the halo electrons bi-Maxwellian distributed and neglects the effects of the core population. Here in Fig. 5 we show that this disagreement may be resolved by a more realistic comparison with the instability thresholds provided by a bi-Kappa approach. The instability threshold for the bi-Maxwellian limit $(\kappa \rightarrow \infty)$ invoked by Štverák et al. (2008) is also displayed for reference with dotted (black) lines. The instability thresholds are markedly lowered with decreasing the power-index $\kappa$ and for low values of $\kappa$ these thresholds approach the limits of the temperature anisotropy observed in the solar wind. The instability thresholds also shape very well the isocontours counting the number of events in the bottom panel. However, we should stress that our comparison in Fig. 5 is based on a similar zero-order approach that minimizes any influence of the core electrons but assumes the suprathermal electrons more realistically described by a bi-Kappa distribution function. 


\section{DISCUSSIONS AND CONCLUSIONS}

In this paper, we have proposed a refined theory of the EFHI in collisionless plasmas with bi-Kappa-distributed electrons, seeking new and valuable evidence for an extended implication of this instability in the relaxation of temperature anisotropy in space plasmas. Our present study is particularly motivated by the solar wind observations that do not confirm the indefinite increase of temperature predicted by the solar wind expansion in the direction parallel to the interplanetary magnetic field, but reveal very clear bounds for the temperature anisotropy of plasma particles. Previous studies have focused on the thermal (core) populations of electrons and protons, developing standard bi-Maxwellian approaches and showing that large deviations from isotropy may be constrained by the kinetic instabilities (Hellinger et al. 2006; Štverák et al. 2008). However, the same bi-Maxwellian is not appropriate to describe suprathermal populations and their anisotropy, and cannot prescribe accurately the resulting instabilities and their back reaction on these populations.

Here we have assumed the anisotropic electrons well reproduced by a bi-Kappa distribution function, which has widely been invoked as a global model to describe the entire distribution, incorporating both the core and halo components (Hellberg et al. 2005; Pierrard \& Lazar 2010). The same bi-Kappa model can also describe only the halo component, i.e., the suprathermal tails of the electron distributions observed in the solar wind (Maksimovic et al. 2005; Štverák et al. 2008). In addition, the Kappa approach considered here assumes a $\kappa$-dependent temperature, thus enabling a realistic interpretation of the Kappa populations and their effects (theoretical and observational arguments are provided in the Introduction). The results of our present study markedly contrast with those provided by Lazar \& Poedts (2009) and Lazar et al. (2011), who studied the same EFHI but driven by bi-Kappa electrons with a $\kappa$-independent temperature. Thus, we have identified two distinct regimes of the EFHI (Section 3), which are differentiated by the wavenumber dispersion laws obtained for the frequency and growth rate. More specific to the EFHI are the unstable modes exemplified in Figs 1 and 2 with frequencies that can significantly exceed the proton cyclotron frequency $\Omega_{\mathrm{p}}$. To establish this regime, the anisotropic electrons must have a significant amount of kinetic free energy, implying electrons with a high plasma beta or/and a large enough anisotropy. If damped, these modes cannot extend above $\Omega_{\mathrm{p}}$ and their wavenumber dispersion keeps the aspect of low-frequency EMIC modes in the absence of kinetic anisotropies. At higher wavenumbers (lower scales), these damped modes can change their polarity converting to the branch of RH-polarized modes (whistlers). These EM modes with a wavenumber dispersion resembling that of the EMIC modes, i.e. with wave-frequency increasing asymptotically to $\Omega_{\mathrm{p}}$, can be destabilized by the EFHI for conditions approaching marginal stability. A few cases relevant for this new regime are presented in Figs 3 and 4, with mention that top panels in these figures include unstable solutions representative for a transition between the two distinct regimes of the EFHI.

The values considered for the plasma parameters in Figs 1-4 are typically encountered in the solar wind, and for these conditions the EFHI develops only in the presence of suprathermal electrons, i.e. for finite values of $\kappa$, while for (bi-)Maxwellian limit $\kappa \rightarrow \infty$ these modes are damped. Increasing the presence of suprathermal populations (by lowering $\kappa$ ) has a non-uniform effect on the wavefrequency of the unstable modes, which highly depends on the regime of FHI, and this becomes evident if we compare for in- stance Figs 2 and 4. Contrary to the previous results in Lazar \& Poedts (2009) and Lazar et al. (2011), involving bi-Kappa electrons with a $\kappa$-independent temperature, here the EFHI is systematically stimulated by the suprathermal electrons, which enhance the (maximum) growth rates for any regime of this instability. This effect is the most pronounced for conditions approaching the marginal stability (Fig. 3), which also explains the significant decrease of the instability thresholds in Fig. 5 for lower values of $\kappa$.

These results provide a straightforward characterization of the EFHI driven by the anisotropic electrons, when their velocity distribution is overall well described by a (global) bi-Kappa distribution function. A natural confirmation of the role played by this instability in constraining the temperature anisotropy of electrons may be obtained from a direct comparison of the instability thresholds with the limits of anisotropy measured in the solar wind. Statistical diagrams of temperature anisotropy versus plasma beta parameter determined with a global bi-Kappa fitting model are not available and a confrontation with our threshold conditions is therefore not possible at this stage. However, such diagrams of statistical data have been produced for the halo component, using the same bi-Kappa fitting model to evaluate the main parameters of this component, e.g. density, temperature, temperature anisotropy, etc. (Štverák et al. 2008). We have used these diagrams in Fig. 5 for a direct comparison of the instability thresholds with the limits of the halo anisotropy reported by the observations, considering the bi-Kappa approach developed here as a zero-order approach of the halo electrons. To keep the analysis straightforward, in this analysis we have isolated only the instability effects of the bi-Kappa electrons, and neglected the effects of the core population. Although the model is simplified, the instability thresholds are in surprisingly good agreement with the observations. Given that solar wind electrons, and especially their suprathermal populations are collisionless, such a good agreement between theory and observations represents a plausible confirmation on the role played by the FHI in constraining the solar wind electron anisotropy. These results should stimulate future studies to refine the instability conditions for an even more realistic approach that includes the effects of a (bi-)Maxwellian core. Such a complex model combining a (bi-)Maxwellian core and a bi-Kappa halo was applied recently for studies of cyclotron instabilities (Lazar, Poedts \& Schlickeiser 2014a; Lazar et al. 2015a; Shaaban et al. 2016b).

To conclude, an agreement between the instability thresholds and the bounds of the temperature anisotropy measured in the solar wind is conditioned by a proper modelling of the velocity distributions in accord to the observations. In this paper, an important progress is made by introducing the bi-Kappa modelling with $\kappa$-dependent temperatures for the anisotropic suprathermal electrons. Our present results strongly suggest that the EFHI may efficiently constrain the temperature anisotropy of the suprathermal electrons in the slow wind, complementing the results by Štverák et al. (2008), which showed similar effects of this instability on the core electrons. This instability can therefore intermediate a transfer of energy between parallel and perpendicular directions. From an extended perspective, we can further claim that the resulting low-frequency fluctuations may also establish an energy transfer from small to large scales, namely from the electrons, mainly from their energetic (suprathermal) populations that carry the main heat flux in the solar wind, to the resonant protons. Although suprathermal populations are not easily captured in numerical experiments, our present results provide valuable premises and motivations for building new and advanced algorithms to confirm these mechanisms. 


\section{ACKNOWLEDGEMENTS}

The authors acknowledge support from the Katholieke Universiteit Leuven, Ruhr-University Bochum, and Alexander von Humboldt Foundation. These results were obtained in the framework of the projects GOA/2015-014 (KU Leuven), G0A2316N (FWO-Vlaanderen), and C 90347 (ESA Prodex 9). The research leading to these results has also received funding from the European Commission's Seventh Framework Programme FP7-PEOPLE2010-IRSES-269299 project-SOLSPANET (www.solspanet.eu). SMS would like to thank the Egyptian Ministry of Higher Education for supporting his research activities.

\section{REFERENCES}

Camporeale E., Burgess D., 2008, J. Geophys. Res., 113, A07107

Chew G. F., Goldberger M. L., Low F. E., 1956, Proc. R. Soc. A, 236, 112

Collier M. R., Hamilton D. C., Gloeckler G., Bochsler P., Sheldon R. B., 1996, Geophys. Res. Lett., 23, 1191

Eviatar A., Schulz M., 1970, Planet. Space Sci., 18, 321

Fried B. D., Conte S. D., 1961, The Plasma Dispersion Function. Academic Press, New York

Gary S. P., 1993, Theory of Space Plasma Microinstabilities. Cambridge Univ. Press, Cambridge

Gary S. P., Lee M. A., 1994, J. Geophys. Res., 99, 11297

Gary S. P., Nishimura K., 2003, Phys. Plasmas, 10, 3571

Gary S. P., Li H., O’Rourke S., Winske D., 1998, J. Geophys. Res., 103, 14567

Hellberg M., Mace R., Cattaert T., 2005, Space Sci. Rev., 121, 127

Hellinger P., Trávníček P. M., Kasper J. C., Lazarus A. J., 2006, Geophys. Res. Lett., 33, L09101

Hellinger P., Trávníček P. M., Decyk V. K., Schriver D., 2014, J. Geophys. Res., 119, 59

Kasper J. C., Lazarus A. J., Gary S. P., 2002, Geophys. Res. Lett., 29, 1839 Lazar M., Poedts S., 2009, A\&A, 494, 311

Lazar M., Schlickeiser R., Shukla P. K., 2008, Phys. Plasmas, 15, 042103

Lazar M., Poedts S., Schlickeiser R., 2011, A\&A, 534, A116
Lazar M., Schlickeiser R., Poedts S., 2012, in Lazar M., ed., Exploring the Solar Wind. InTech, Rijeka, Croatia, p. 241. Available at: http://www.intechopen.com/books/exploring-the-solar-wind/

Lazar M., Poedts S., Schlickeiser R., 2014a, J. Geophys. Res., 119, 9395

Lazar M., Poedts S., Schlickeiser R., Ibscher D., 2014b, Sol. Phys., 289, 369

Lazar M., Poedts S., Schlickeiser R., Dumitrache C., 2015a, MNRAS, 446, 3022

Lazar M., Poedts S., Fichtner H., 2015b, A\&A, 582, A124

Lazar M., Fichtner H., Yoon P. H., 2016, A\&A, 589, A39

Leubner M., 2004, ApJ, 604, 469

Leubner M., Schupfer N., 2000, J. Geophys. Res., 105, 27387

Leubner M., Schupfer N., 2001, J. Geophys. Res., 106, 12993

Lin R. P., 1998, Space Sci. Rev., 86, 61.

Maksimovic M., Pierrar V., Riley P., 1997, Geophys. Res. Lett., 24, 1151

Maksimovic M. et al., 2005, J. Geophys. Res., 110, A09104

Messmer P., 2002, A\&A, 382, 301

Meyer-Vernet N., 2007, Basic of the Solar Wind. Cambridge Univ. Press, Cambridge, p. 266

Newbury J. A., Russell C. T., Phillips J. L., Gary S. P., 1998, J. Geophys. Res., 103, 9553

Paesold G., Benz A. O., 1999, A\&A, 351, 741

Pierrard V., Lazar M., 2010, Sol. Phys., 267, 153

Pierrard V., Lazar M., Štverák Š., Poedts S., Maksimovic M., Trávníček P., 2016, Sol. Phys., 291, 2165

Rosenbauer H. et al., 1977, J. Geophys., 42, 561

Shaaban S. M., Lazar M., Poedts S., Elhanbaly A., 2016a, J. Geophys. Res., 121,6031

Shaaban S. M., Lazar M., Poedts S., Elhanbaly A., 2016b, Astrophys. Space Sci., 361, 1

Štverák Š., Trávníček P., Maksimovic M., Marsch E., Fazakerley A. N., Scime E. E., 2008, J. Geophys. Res., 113, A03103

Vasyliunas V. M., 1968, J. Geophys. Res., 73, 2839

Viňas A. F., Moya P. S., Navarro R. E., Valdivia J. A., Araneda J. A., MuÃśoz V., 2015, J. Geophys. Res., 120, 3307

This paper has been typeset from a $\mathrm{T}_{\mathrm{E}} \mathrm{X} / \mathrm{LT} \mathrm{E} \mathrm{X}$ file prepared by the author. 